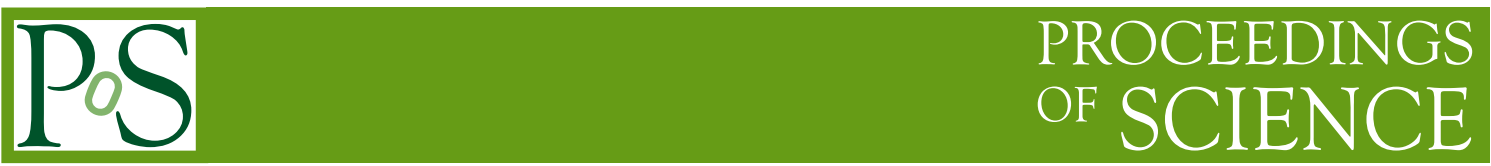

\title{
KATRIN: an experiment to determine the neutrino mass
}

\author{
Florian FRAENKLE* \\ Karlsruhe Institute of Technology \\ E-mail: florian.fraenkleakit.edu
}

\section{KATRIN Collaboration}

The KArlsruhe TRItium Neutrino (KATRIN) experiment is a next generation, model independent, large scale experiment to determine the mass of the electron anti-neutrino by investigating the kinematics of tritium $\beta$-decay with a sensitivity of $0.2 \mathrm{eV} / \mathrm{c}^{2}$. The measurement setup consists of a high luminosity windowless gaseous molecular tritium source (WGTS), a differential and cryogenic pumped electron transport and tritium retention section, a tandem spectrometer section (pre-spectrometer and main spectrometer) for energy analysis, followed by a detector system for counting transmitted $\beta$-decay electrons. In order to achieve the desired sensitivity the WGTS, in which tritium decays with an activity of about $10^{11} \mathrm{~Bq}$, needs to be stable on the $0.1 \%$ level in injection pressure and temperature at an absolute value of $30 \mathrm{~K}$. With the capability to create an axial magnetic field of 3.6 T the WGTS is going to be one of the world's most complex superconducting magnet and cryostat systems. The Main Spectrometer (length $24 \mathrm{~m}$, diameter $10 \mathrm{~m}$ ), which works as a retarding electrostatic spectrometer, will have an energy resolution of $0.93 \mathrm{eV}$ at $18.6 \mathrm{keV}$. For the precise energy analysis at the tritium endpoint, a retarding potential of $-18.6 \mathrm{kV}$ is needed with $1 \mathrm{ppm}$ stability. In order to reach the background level needed to achieve the sensitivity, it will be operated at a pressure of $10^{-11}$ mbar. This report will give an overview of the actual status of the project.

European Physical Society Europhysics Conference on High Energy Physics

July 16-22, 2009

Krakow, Poland

* Speaker. 


\section{Introduction}

Neutrinos play an important role in the evolution of the universe. Due to their large abundance (the neutrino density of the universe is about $330 \mathrm{v}$ s $/ \mathrm{cm}^{3}$ for all three flavours) they can affect the evolution of large scale structures depending on their mass. Neutrino oscillation experiments showed that neutrinos are massive and could set a lower limit of $40 \mathrm{meV} / \mathrm{c}^{2}$ [1] on the neutrino mass. Experiments investigating the kinematics of $\beta$-decay set an upper limit on the neutrino mass of $2.3 \mathrm{eV} / \mathrm{c}^{2}$ [2]. There are recent attempts to determine the absolute neutrino mass using data from cosmology to improve the upper limit. Anyhow, the limit depends strongly on the data and model that is used. The KATRIN experiment uses a model independent method to determine the mass of the electron anti-neutrino $\left(m_{\bar{v}_{e}}\right)$ by investigating the kinematics of tritium $\beta$-decay. With a sensitivity of $0.2 \mathrm{eV} / \mathrm{c}^{2}$ it has the potential to probe the mass range of $m_{\bar{v}_{e}}$ where neutrinos have a significant influence on structure formation in the universe.

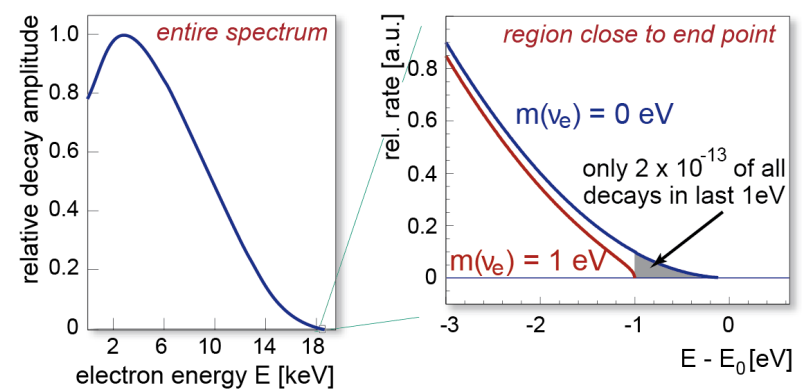

Figure 1:

energy spectrum of tritium $\beta$-decay

In $\beta$-decay a neutron in the nuclei decays into a proton and releases an electron $\left(e^{-}\right)$and a electron anti-neutrino $\left(\bar{v}_{e}\right)$. The energy released in the decay is distributed to $e^{-}$and $\bar{v}_{e}$ in a statistical fashion. The energy spectra of the electron is given by the Fermi theory of $\beta$-decay [3] (see equation 1.1). $m_{\bar{v}_{e}}^{2}$ enters as a parameter and its effect on the shape of the spectrum is large close to the end point $\left(E_{0}\right)$. The right side of figure 1 shows the energy spectrum of $\beta$ electrons for the case of $m_{\bar{v}_{e}}=0 \mathrm{eV} / \mathrm{c}^{2}$ and $m_{\bar{v}_{e}}=1 \mathrm{eV} / \mathrm{c}^{2}$. In KATRIN $m_{\bar{v}_{e}}^{2}$ will be determined by measuring the shape of the $\beta$-spectrum close to its endpoint.

$$
\frac{d N}{d E}=\frac{G_{F}^{2}}{2 \pi^{3}} \cos ^{2} \Theta_{C}|M|^{2} F(Z, E) p\left(E+m_{e} c^{2}\right)\left(E_{0}-E\right) \sqrt{\left(E_{0}-E\right)^{2}-m_{\bar{v}_{e}}^{2} c^{4}}
$$

\section{The KATRIN Experiment}

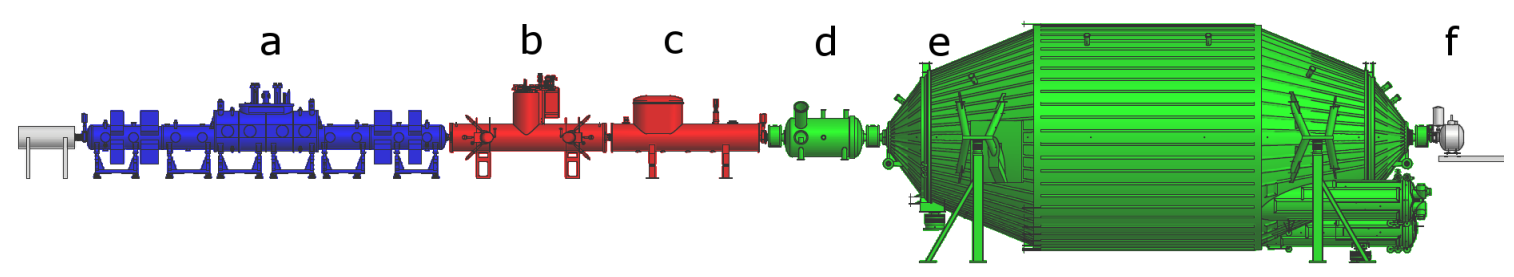

Figure 2: schematic overview of the KATRIN experiment setup

The KATRIN experiment [4] consists of six major subsystems (see figure 2): a windowless gaseous molecular tritium source (WGTS), b differential pumping section (DPS), $\mathbf{c}$ cryogenic pumping section (CPS), $\mathbf{d}$ pre-spectrometer, e main spectrometer and $\mathbf{f}$ detector system. 
a WGTS: The tritium source consists of a $10 \mathrm{~m}$ long tube with a diameter of $90 \mathrm{~mm}$ and is operated at a temperature of $30 \mathrm{~K}$ by circulation of two phase Neon. Tritium $\left(\mathrm{T}_{2}\right)$ is injected into the centre of the tube and decays with a rate of $10^{11} \mathrm{~Bq}$ to provide a sufficient number of electrons close to $E_{0}$ (see fig. 1). The electrons are guided via an axial magnetic field of 3.6 $\mathrm{T}$ towards the spectrometers. The $\mathrm{T}_{2}$ is collected via turbomolecular pumps at the end of the WGTS and recirculated via an "inner loop" which removes contaminants (particularly ${ }^{3} \mathrm{He}$ ) and is capable to process $40 \mathrm{~g} \mathrm{~T}_{2}$ a day.

Status: A demonstrator to verify the stringent thermal performance specifications (temperature stability $\pm 30 \mathrm{mK}$ ) will be delivered in early 2010 . The WGTS itself will be manufactured in 2011/12.

b DPS: The task of the DPS is to reduce the $T_{2}$ partial pressure by a factor of $>10^{5}$ and to guide the electrons via a strong magnetic field of 5.6 T. The beam tube has four bends to avoid beaming of $\mathrm{T}_{2}$ molecules towards the spectrometers and to increase the pumping power of the turbomolecular pumps attached there.

Status: The DPS has been delivered in July 2009. Acceptance tests are ongoing and a test program to investigate the gas reduction factor and the electron transport properties is planned for 2010 .

c CPS: Any remaining $\mathrm{T}_{2}$ that passes the DPS is trapped in the CPS in Argon frost frozen on the $4 \mathrm{~K}$ cold beam tube, which forms a highly efficient, large-area and radiation-immune surface. A refreshment cycle of the Argon frost is needed every 2 months.

Status: The technical design report is finished and the CPS is currently being manufactured.

d Pre-spectrometer: The pre-spectrometer as well as the main spectrometer are MAC-E filters [5] (Magnetic Adiabatic Collimation combined with an Electrostatic Filter). In the final setup the pre-spectrometer will be used as a pre-filter on a potential a few hundred volts below $\mathrm{E}_{0}$. Status: Until the end of 2009 the pre-spectrometer is operated in a test setup, for more details see section 3 .

e Main spectrometer: The purpose of the $10 \mathrm{~m}$ in diameter and $24 \mathrm{~m}$ long main spectrometer is the energy analysis of the $\beta$-decay electrons. It has an energy resolution of $0.93 \mathrm{eV}$ at $18.6 \mathrm{keV}$. In order to achieve the desired background rate of $<10 \mathrm{mHz}$ a double layer inner electrode system made of thin wires - mounted with submillimeter precision - is installed. The wire layers are put on a more negative potential (100/200 V) with respect to the tank voltage in order to shield secondary electrons produced in the vessel wall by cosmic particles. The absolute voltage of $-18.6 \mathrm{kV}$ needs to be stable on the $1 \mathrm{ppm}$ level and is monitored with a high precision voltage divider [6] and an independent monitoring and calibration beam line.

Status: Currently the inner wire electrode system is being mounted. Electro-magnetic test measurements will start in 2010.

f Detector: Electrons that are able to overcome the potential barriers of the spectrometers are detected in a monolithic 148 pixel silicon PIN diode array $100 \mathrm{~mm}$ in diameter. Selection of materials, shielding and an active veto are used to keep the intrinsic detector background below $1 \mathrm{mHz}$. 
Status: Currently under construction, assembly and initial commissioning are planned for mid of 2010.

\section{Pre-spectrometer Test Setup}

The test setup consists of the pre-spectrometer itself, an ultra high vacuum recipient including an inner electrode system, two super-conducting solenoids (max. $4.5 \mathrm{~T}$ ), an photoelectric electron source (e-gun) attached at one side and a detector chamber housing a monolithic 64 pixel silicon PIN diode on the other side. The test setup acts as a prototype for the main spectrometer in order to test the vacuum concept and the new electro-magnetic design of the KATRIN spectrometers. In addition new technologies are tested and developed at the test setup which will be applied to KATRIN e.g. an active HV stabilization, data acquisition system and background suppression.

In the recent past the main focus at the test setup was to optimize the geometry of the electrode system at the entrance region of the pre-spectrometer. Due to high magnetic and electric fields inside the KATRIN spectrometers it is possible to create Penning traps there. By scattering of trapped particles on residual gas molecules more trapped particles can be created and this process can lead to a penning discharge which can significantly increase the background. It was observed at the pre-spectrometer test setup that a small Penning trap on the mm scale can cause a significant increase in background rate up to several $\mathrm{kHz}$.

To avoid all Penning traps in the pre-spectrometer a special shaped ground electrode was developed. The electro-magnetic properties of the new electrode have been simulated in detail and the electrode was manufactured of aluminium with high precision. After mounting the new electrode in summer 2009 first measurements showed that the spectrometer now runs almost background free (rate $<100 \mathrm{mHz})$ at the design parameter of retarding potential $(-18 \mathrm{kV})$ and magnetic field $(4.5 \mathrm{~T})$. As a result the design of the ground electrode is being adopted to the ground electrode of the main spectrometer.

Until end of 2009 a detailed measurement program is planned to investigate the background performance in more detail as well as studies of the transmission characteristics of the pre-spectrometer.

\section{References}

[1] C. Amsler et al. (Particle Data Group), Physics Letters B667, 1 (2008)

[2] Kraus C. et al. Final Results from phase II of the Mainz Neutrino Mass Search in Tritium $\beta$ Decay, Eur. Phys. J. C40 447-468 2005 [arXiv:hep-ex/0412056]

[3] E. Fermi, Versuch einer Theorie der $\beta$-Strahlen, Zeitschrift f. Physik $\mathbf{A 8 8}$ 161-177 (1934)

[4] KATRIN Collaboration, KATRIN Design Report 2004, FZKA 7090 [http://bibliothek.fzk.de/zb/berichte/FZKA7090.pdf]

[5] G. Beamson, U. Porter, D. Turner, The collimating and magnifying properties of a superconducting field photoelectron spectrometer, Journal of Physics E: Scientific Instruments 13 (64-66) 1980

[6] Th. Thümmler, R. Marx, Ch. Weinheimer, Precision high voltage divider for the KATRIN experiment Preprint [arXiv:0908.1523v1] 